\title{
Optimal Placement of a Superior Vena Cava Cannula in Minimally Invasive Robot-Assisted Cardiac Surgery
}

\author{
Yoon Kyung Lee, MD; Ji Yeon Sim, MD; Jung Wook Seo, MD*; \\ In Cheol Choi, MD; Kyung Don Hahm, MD; Jae Woong Choi, MD
}

\begin{abstract}
Background: Minimally invasive robot-assisted cardiac surgery is generally performed nowadays. To avoid the interference of a superior vena cava (SVC) cannula during surgery, it should be inserted before the operation. The position of this cannula is very important because it can cause poor venous drainage during operation. The proper position of the SVC cannula was investigated in the present study.
\end{abstract}

\begin{abstract}
Methods and Results: The position of the SVC cannula using the transesophageal echocardiography (TEE) and chest X-ray in 45 patients was ascertained. The distances from the SVC cannula tip to the carina, sternal end of the right clavicle and the lower margin of the T4 vertebral body on chest X-rays were measured. The mean depth of the SVC cannula was $142.0 \pm 11.6 \mathrm{~mm}$. The correlation coefficients of cannula depth with sex, weight and height were $0.519,0.399$ and 0.382 , respectively.
\end{abstract}

Conclusions: The appropriate depth of an SVC cannula has weakly positive relationships with sex, weight and height. The results of the present study suggest that chest X-rays might be necessary to confirm the appropriate location of the cannula and that TEE might be the method of choice for correct positioning of the SVC cannula in minimally invasive robot-assisted cardiac surgery. (Circ J 2010; 74: 284-288)

Key Words: Catheterization; Robot-assisted cardiac surgery; Superior vena cava

$\mathbf{O}$ pen heart surgery still has the highest morbidity and mortality rates compared with other surgeries, such as major vascular or neurosurgery. To make smaller surgical incision is a desirable goal, and different strategies and approaches have been attempted to achieve this.

Nowadays, mini-thoracotomy is generally used because it can reduce invasiveness, the risk of infection and postoperative pain. ${ }^{1}$ In mini-thoracotomy, the incision $(4 \mathrm{~cm}$ in size) is performed at the fourth intercostal space (ICS), automated endoscope for optimal positioning (AESOP) is inserted at the third ICS and transthoracic aortic cross-clamp is followed via the second ICS. It is necessary to insert a superior vena cava (SVC) cannula via internal jugular vein percutaneously. If the SVC cannula is too deep, it would be an obstacle to the operation and might cause venous air lock in the bypass circuit. In the case of shallow SVC cannula, the risk of microemboli ${ }^{2}$ and blood trauma ${ }^{3}$ would be increased. The optimal position of the SVC cannula and the SVC-right atrial (RA) junction, is important for adequate venous drainage.

Despite this fact, there have been no useful guidelines on the optimal depth of the SVC cannula in patients undergoing minimal cardiovascular surgery. Therefore, the current study was conducted to find a confirmative method to identify the depth of the jugular puncture site to the SVC-RA junction by examining the relationship between the depth of the SVC cannula and anthropometric variables and also by investigating the proper position of the SVC cannula using transesophageal echocardiography and chest X-rays.

\section{Methods}

The Institutional Review Board of our hospital approved our study protocol including informed consent. Forty-five patients (aged 43.2 \pm 14.2 years) scheduled to have AESOP-guided

\begin{tabular}{|lcc|}
\hline \multicolumn{3}{|c|}{ Table 1. Demographic Data } \\
& Male $(\mathbf{n}=\mathbf{1 6})$ & Female $(\mathbf{n}=\mathbf{2 6})$ \\
Age (years) & $41.6 \pm 9.4$ & $44.6 \pm 16.6$ \\
Weight $(\mathrm{kg})$ & $69.7 \pm 7.2$ & $53.3 \pm 6.1$ \\
Height $(\mathrm{cm})$ & $171.7 \pm 5.9$ & $157.7 \pm 6.1$ \\
Body mass index $\left(\mathrm{kg} / \mathrm{m}^{2}\right)$ & $23.8 \pm 2.4$ & $21.4 \pm 3.0$ \\
\hline
\end{tabular}

Values are presented as mean $\pm \mathrm{SD}$.

Received August 17, 2009; revised manuscript received October 6, 2009; accepted October 7, 2009; released online December 22, 2009 Time for primary review: 43 days

Department of Anesthesiology and Pain Medicine, Asan Medical Center, University of Ulsan College of Medicine, Seoul, *Department of Diagnostic Radiology, Ilsan Paik Hospital, Inje University School of Medicine, Goyang, Gyeonggi, Korea

Mailing address: Ji Yeon Sim, MD, Department of Anesthesiology and Pain Medicine, Asan Medical Center, College of Medicine, University of Ulsan, 388-1 Pungnap-2dong, Songpa-gu, Seoul 138-736, Korea. E-mail: jysim@amc.seoul.kr

ISSN-1346-9843 doi:10.1253/circj.CJ-09-0600

All rights are reserved to the Japanese Circulation Society. For permissions, please e-mail: cj@j-circ.or.jp 


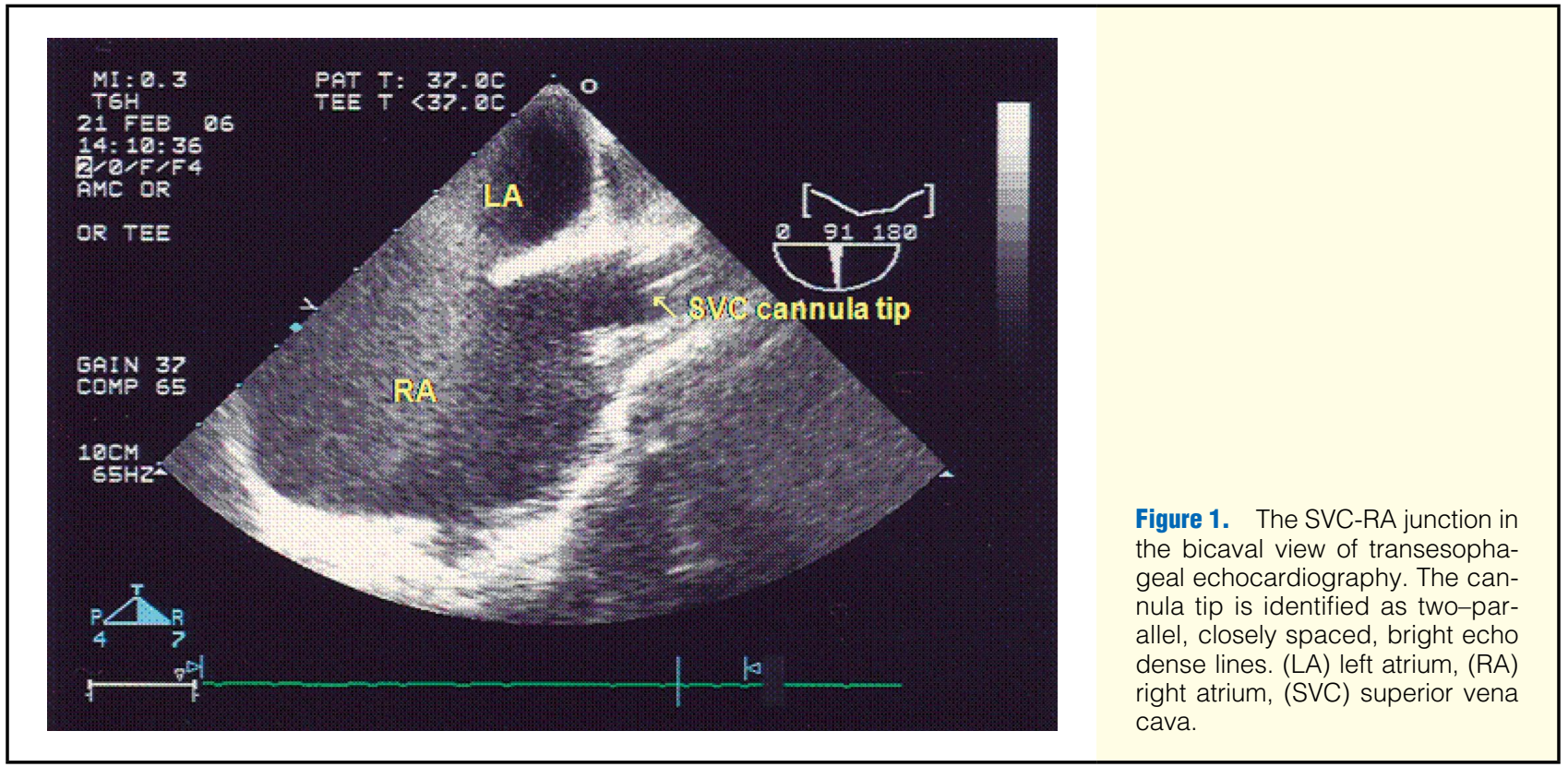

cardiac surgery were included (Table 1). Three patients were excluded because 1 had a small French cannula (17 Fr) as a result of a small-sized SVC and 2 had a left-sided cannula because of hematoma on the right side. Exclusion criteria were contraindications to transesophageal echocardiography (TEE) (eg, esophageal pathology or surgery), cardiac arrythmias other than sinus arrythmia and preoperative cardiomegaly determined by transthoracic echocardiography.

\section{SVC Cannulation}

After induction of general anesthesia, patients were placed in a supine $20^{\circ}$ Trendelenburg position. After turning the head $45^{\circ}$ to the left, the right internal jugular vein was punctured at the point $4 \mathrm{~cm}$ from the upper margin of the clavicle, which was located in the triangle formed by the clavicle, and the sternal and clavicular heads of the sternocleidomastoid muscle. After confirming free withdrawal of fresh venous blood with a 5-ml spring-wire introduction syringe (Arrow raulerson, Arrow international, Reading, PA, USA) we performed the sterile Seldinger technique. A tissue dilator was inserted through the spring wire. Skin incision was made in order to insert a 21 Fr cannula (DLP cannula, Medtronic, Minneapolis, MN, USA). The femoral vein was selected for the cannula insertion site in order to drain inferior vena caval blood. Arterial cannulation was also performed at the site of the femoral artery. During the main procedure, we performed one-lung ventilation using a left-sided double lumen endotracheal tube.

\section{Transesophageal Echocardiography}

Before draping the cannulation site, a TEE transducer $(6.2 \mathrm{MHz}$ phased multiplane probe/Sonos 5500, Philips, Bothell, WA, USA) was inserted to a mid-esophageal position and rotated in a clockwise direction. The interatrial septum was identified at a depth of $10 \mathrm{~cm}$ on the mid-esophageal bicaval view by published guidelines. ${ }^{4}$ During insertion of the SVC cannula via the right internal jugular vein, the first assistant managed the plane of the probe at an angle of $90-110^{\circ}$ to produce a bicaval view for the optimal positioning of the SVC cannula at the SVC-RA junction. The echocardiographic

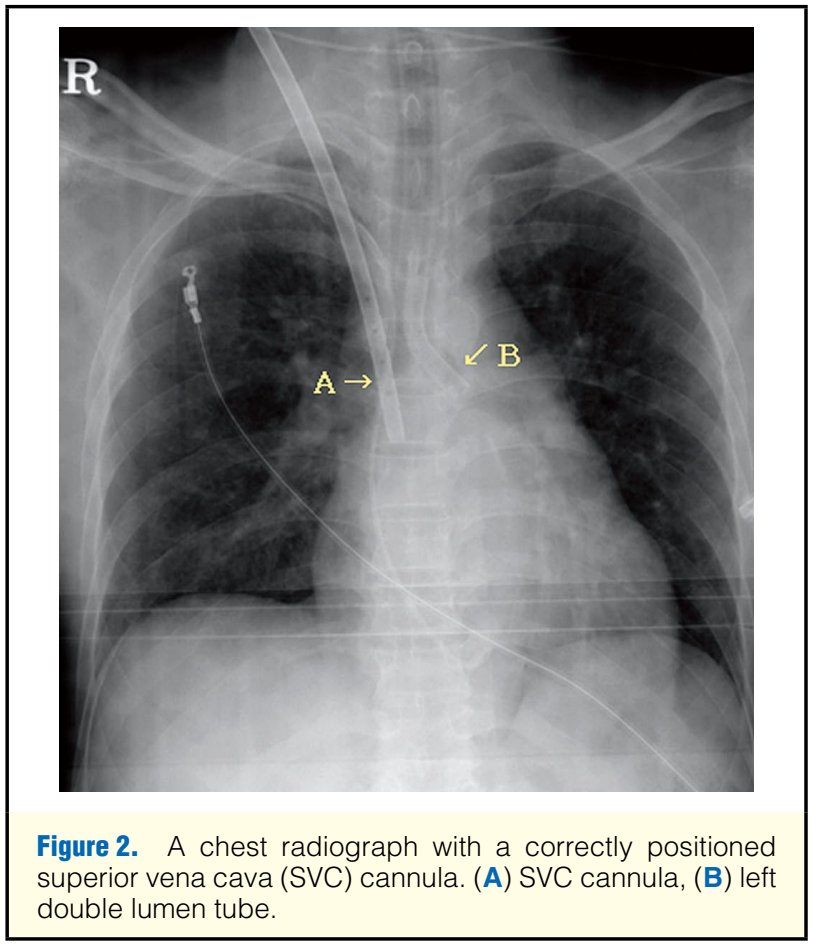

correlate of the SVC-RA junction was defined as the base of the superior edge of the crista terminalis. A rapid flush of $10 \mathrm{ml}$ of physiological saline containing 200 IU of heparin was used to identify the SVC cannula tip. The microbubbles of the solution acted as a contrast medium and helped to identify the plane of the SVC in which the catheter tip is located. The catheter tip was usually identified as two parallel, closely spaced, bright echo dense lines (Figure 1).

\section{Chest X-Ray}

After TEE insertion, all chest radiographs were taken as a portable anteroposterior chest X-rays with patients lying in 
Table 2. The Correlation Between the Insertion Depth of SVC Cannula and Each Parameter

\begin{tabular}{|c|c|c|}
\hline Factor & Correlation coefficients $(r)$ & P-value \\
\hline Sex & 0.519 & 0.0000 \\
\hline Hand length & 0.442 & 0.00355 \\
\hline Foot length & 0.442 & 0.00555 \\
\hline Neck circumference & 0.402 & 0.00853 \\
\hline Weight & 0.399 & 0.00892 \\
\hline Height & 0.382 & 0.0126 \\
\hline LVIDd (diastolic internal diameter of left ventricle) & 0.386 & 0.0116 \\
\hline LVIDs (systolic internal diameter of left ventricle) & 0.330 & 0.0331 \\
\hline Sternal notch to ipsilateral lower margin of ear lobe & 0.328 & 0.0338 \\
\hline SVC cannula tip to lower margin of T4 vertebral body & 0.309 & 0.0466 \\
\hline SVC cannula tip to sternal end of right clavicle & 0.306 & 0.049 \\
\hline Sternal notch to nipple line & 0.298 & 0.0554 \\
\hline
\end{tabular}

The insertion depth of SVC cannula was plotted against with each parameter.

SVC; superior vena cava.

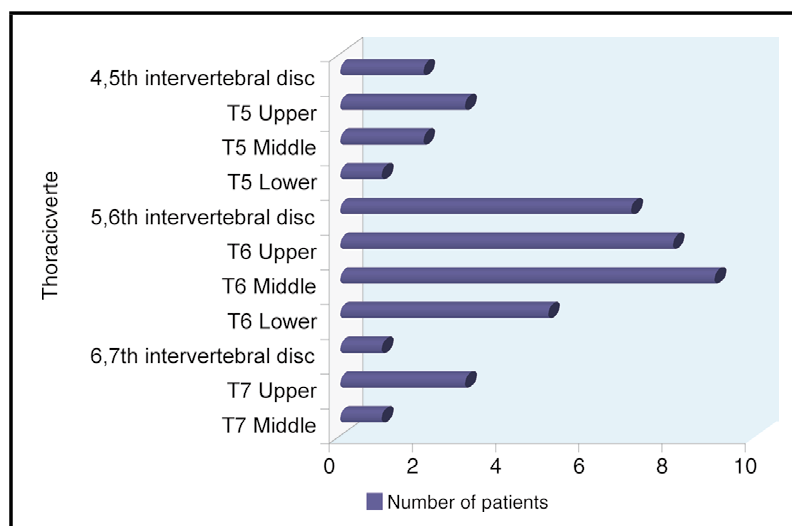

Figure 3. The location of a superior vena cava cannula tip. It is mainly located at T5-6 intervertebral disc space, and the T6.

the supine position. Because the radiographic SVC-RA junction was usually not clearly seen, we used several landmarks to assess the location of the SVC cannula. The patient archiving and communication system (PACS; New Petavision for Clinics, Seoul, Korea) was used to visualize the digital images of the chest radiographs. ${ }^{5} \mathrm{We}$ measured the distances from the SVC cannula tip to the carina, to the sternal end of the right clavicle and the lower margin of T4 vertebral body (VB) on chest X-rays. We also measured authropometric variables such as neck circumference, hand length, foot length and the distance from the sternal notch to the ipsilateral nipple, to the lower margin of the ipsilateral ear lobe and to the ipsilateral mandible angle (Figure 2). The correlation of SVC cannula insertion depth with each factor was statistically assessed by using Sigma Stat (9.0, Sigma plot 2000; SPSS, San Jose, CA, USA). The descriptive data are expressed as mean \pm SD. The raw data were summarized as percentages, means and range. Statistical significance was defined as a P-value of less than 0.05 .

\section{Results}

The mean insertional depth of the SVC cannula was 142.2 \pm $11.6 \mathrm{~mm}$. The correlation coefficients of cannula depth with sex, height and weight were $0.519,0.399$ and 0.382 , respec- tively. The correlation coefficients of cannula depth with neck circumference, hand length, foot length, the distance from the sternal notch to the ipsilateral lower margin of the ear lobe and the distance from the sternal notch to the nipple line were $0.402,0.442,0.442,0.328$ and 0.298 , respectively (Table 2). On chest X-rays, the correlation coefficients of cannula depth with the sternal end of right clavicle and the lower margin of T4 VB was 0.306 and 0.309 , respectively (Table 2). However, cannula depth did not correlate with age, the distance from the sternal notch to the ipsilateral mandible, the distance from the SVC cannula tip to the carina and the distance from the SVC cannula tip to the upper margin of the origin of the right upper lobe bronchus. The cannula tip was located at T5-6 intervertebral disc space (IVDS) in $16.7 \%$ of the patients, T6 in $52.4 \%$ of the patients and T6-7 IVDS in $2.4 \%$ of the patients (Figure 3 ). Preoperative echocardiographic findings, such as systolic left ventricular internal diameter and diastolic left ventricular internal diameter, also had positive relationships with insertion depth $(r=0.330$, $\mathrm{r}=0.386$ ).

\section{Discussion}

Due to the straightness of an SVC cannula, the best site for SVC cannulation is the right internal jugular vein. The most important concern of SVC cannulation is the correct placement of the cannula tip with minimal complications, which can be achieved by confirming the cannula site with objective methods and the involvement of an experienced surgeon.

The standard chest X-ray is typically used to confirm the correct positioning of the venous catheter. Different radiographic landmarks for safe positioning have been described. Greenall et al suggested that the tip of the central venous catheter should not be placed more than $2 \mathrm{~cm}$ inferior to a line drawn between the lower borders of the medial ends of the clavicles on an erect postero-anterior chest X-ray. ${ }^{6}$ However, X-ray film is located much closer to the beam source on an anteroposterior chest X-ray taken with a patient lying supine than in postero-anterior chest X-ray taken with a patient standing upright. As a result, anatomical structures located more anteriorly and more peripherally, such as the clavicles, can be augmented on the film by more than $20 \%$. This parallax effect makes the criteria suggested by Greenall less reliable. The carina is located centrally and near the SVC to limit any parallax effect. Caruso et al have demonstrated 


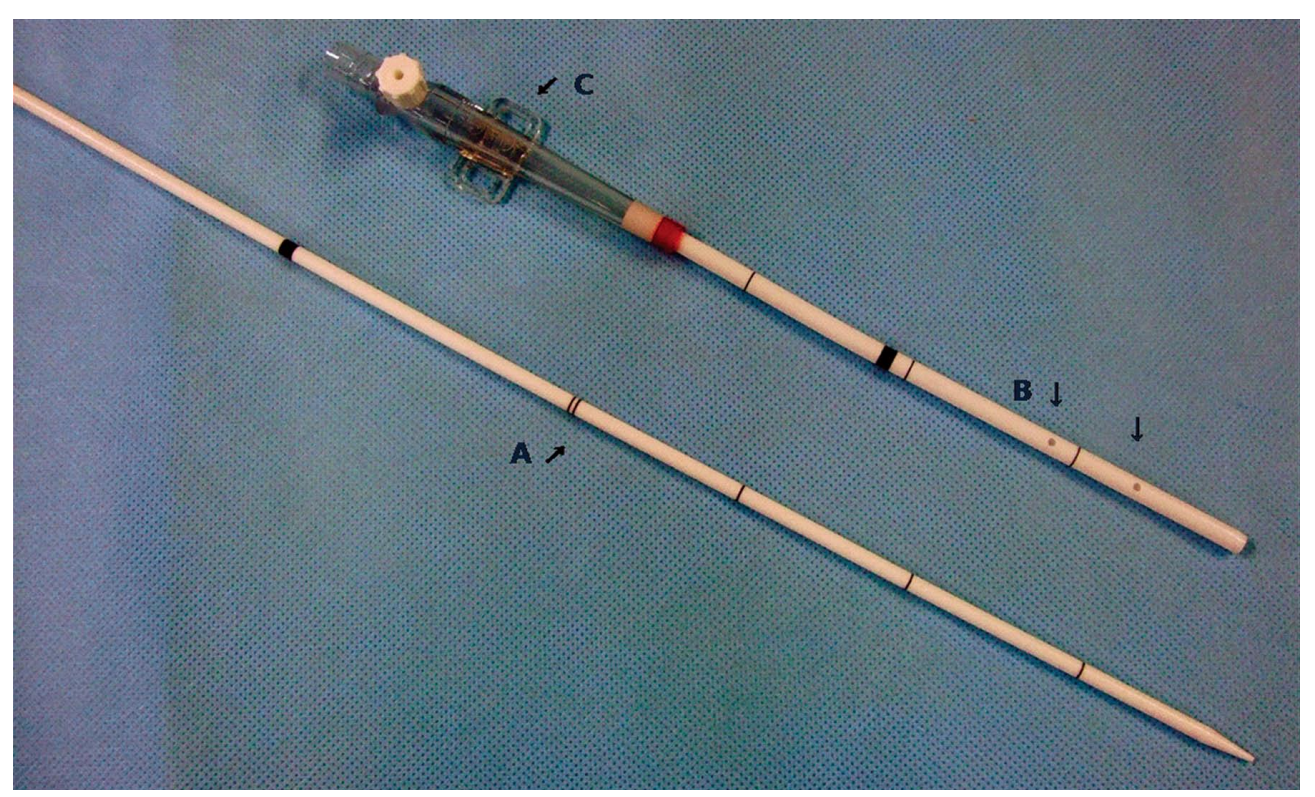

Figure 4. A superior vena cava cannula. (A) Introducer, (B) multiple holes in the cannula tip, (C) a hole for suture to the skin.

that the distal half of the SVC lies within the pericardial reflection and that the midpoint of the SVC is approximately $1.3 \mathrm{~cm}$ above the carina. ${ }^{7}$ It corresponds to the upper limit of pericardial reflection approximately at the level of the takeoff of the right mainstem bronchus as seen on chest X-ray. However, in our study, the carina and the takeoff of the mainstem bronchus had no significant correlation with the insertion depth of the SVC cannula. This might be explained by the fact that the proximal portion of the SVC was not clearly visible on chest antero-posterior X-rays during operation and that the right heart border was changed by cardiac pathology and pulmonary reactivity. In particular, tricuspid regurgitation with pulmonary hypertension might have enlarged the right heart, which was confused with the SVCRA junction.

In the present study, it was found that the depth of the SVC cannula had a weak positive relationship with sex, weight, height, neck circumference, hand length or foot length in patients undergoing AESOP-guided cardiac surgery. Hayashi et al established a simple guideline for determining optimal catheter placement according to the regression line between the catheter depth and height in pediatric cardiac patients. ${ }^{8}$ Height is not affected by cachexia or obesity. However, in their study, the participants were pediatric patients and the position of the catheter tip showed a wide distribution from T1 to T7. In our study, the correlation coefficient of height with the depth of the central line was lower than that of their study. ${ }^{9}$

Connolly et al investigated the landmark for the SVC-RA junction for central venous catheter placement in children by fluoroscopy. ${ }^{10}$ They found that there was a significant correlation with the VB level in all 56 pediatric patients. It was found that the SVC-RA junction was at the level of the T6 or one of its adjacent IVDSs in $92 \%$ of all patients. This is similar to our result: $71.5 \%$ of the cannula tips were located at the level of the T6 or its adjacent IVDSs. It is conceivable that the VBs and IVDS are visible, easy-to-use, and moder- ately accurate landmarks on chest X-ray.

The major problem with correct placement of an SVC cannula is the lack of reliable surface landmarks. Although some authors recommend points such as the sternal angle as being at the level of the junction of the SVC and right atrium, ${ }^{11}$ we did not find any surface landmarks whose correlation coefficients were higher than 0.35. Most investigators recommend a chest X-ray as the only practical, reliable test; however, the depth of safe insertion into the SVC varies among patients and cannot be reliably predicted from external landmarks.

TEE can be used efficiently during intraoperative periods as well as postoperative evaluation. ${ }^{12}$ Based on our results, it is thought that TEE is more accurate than chest X-ray and is adequate for the evaluation of the position of an SVC cannula. We could fix the cannula tightly in order to prevent accidental pullout and to lessen the migration of the initial location in most cases. Taking a chest X-ray after fixing the cannula, we could easily find both the atrium and the SVC on the bicaval view. This technique showed the distinct SVC-RA junction structures. The cannula tip was identified as two parallel, closely spaced, bright echo dense lines. The Chiari network was different from the cannula tip because it was mobile and attached to the Eustachian valve from the IVC side. Heparin solution was used not only to prevent clots in the SVC cannula but also to confirm the tip of the SVC cannula as being agitated.

To achieve optimal drainage from the SVC, we recommend the cannula with multiple holes instead of a single hole at its end (Figure 4). In cases with somewhat deep or shallow locations, it will provide a safety margin as well as good drainage of venous blood.

The results of the present study are subjected to some limitations. First, our results might be influenced by the slight positional changes during the operation. We performed TEE in the $20^{\circ}$ Trendelenburg position with the patient's head being turned $45^{\circ}$ to the left side. Chest X-ray was taken in the 
supine position without head tilting or bed inclination. The operation was performed in the slight semi-lateral position without head tilting or bed inclination. Second, the pallalux of intraoperative chest X-rays might be present in a messy operating room. Thus, we asked one trained technician in radiology to take chest X-rays for the present study.

In conclusion, TEE is the gold standard for correct placement of the SVC cannula during minimally invasive robotassisted cardiac surgery, as it provides real time position and can detect any displacement of cannula during surgery.

\section{References}

1. Je HG, Kim JW, Jung SH, Lee JW. Minimally invasive surgical release of entrapped mapping catheter in the mitral valve. Circ $J$ 2008; 72: $1378-1380$

2. Win KN, Wang S, Undar A. Microemboli generation, detection and characterization during $\mathrm{CPB}$ procedures in neonates, infants, and small children. ASAIO J 2008; 54: 486-490.

3. Martin R, McKenty S, Thisdale Y, Lavallee P, Teijeira J, Bonneau $\mathrm{D}$, et al. Hemolysis during cardiopulmonary bypass. J Cardiothorac Anesth 1989; 3: 737-740.

4. Shanewise JS, Cheung AT, Aronson S, Stewart WJ, Weiss RL, Mark JB, et al. ASE/SCA guidelines for performing a comprehensive intraoperative multiplane transesophageal echocardiography examination: Recommendations of the American Society of Echocardiography Council for Intraoperative Echocardiography and the
Society of Cardiovascular Anesthesiologists Task Force for Certification in Perioperative Transesophageal Echocardiography. Anesth Analg 1999; 89: 870-884.

5. Schummer W, Schummer C, Muller A, Steenbeck J, Fuchs J, Bredle $\mathrm{D}$, et al. ECG-guided central venous catheter positioning: Does it detect the pericardial reflection rather than the right atrium? Eur $J$ Anaesthesiol 2004; 21: 600-605.

6. Greenall MJ, Blewitt RW, McMahon MJ. Cardiac tamponade and central venous catheters. Br Med J 1975; 2: 595-597.

7. Caruso LJ, Gravenstein N, Layon AJ, Peters K, Gabrielli A. A better landmark for positioning a central venous catheter. J Clin Monit Comput 2002; 17: 331-334.

8. Hayashi Y, Maruyama K, Takaki O, Yamauchi J, Ohnishi Y, Kuro M. Optimal placement of CVP catheter in paediatric cardiac patients. Can J Anaesth 1995; 42: 479-482.

9. Kim JH, Kim CS, Bahk JH, Cha KJ, Park YS, Jeon YT, et al. The optimal depth of central venous catheter for infants less than $5 \mathrm{~kg}$. Anesth Analg 2005; 101: 1301-1303.

10. Connolly B, Mawson JB, MacDonald CE, Chait P, Mikailian H. Fluoroscopic landmark for SVC-RA junction for central venous catheter placement in children. Pediatr Radiol 2000; 30: 692-695.

11. Fletcher SJ, Bodenham AR. Safe placement of central venous catheters: Where should the tip of the catheter lie? Br J Anaesth 2000; 85: $188-191$.

12. Kitamura S, Nakatani T, Kato T, Yanase M, Kobayashi J, Nakajima $\mathrm{H}$, et al. Hemodynamic and echocardiographic evaluation of orthotopic heart transplantation with the modified bicaval anastomosis technique. Circ J 2009; 73: 1235-1239. 